\title{
Monitoramento dos pontos críticos de controle aplicado no processamento do leite longa vida em indústria de laticínios de Ji-Paraná
}

\author{
Gleicy Gomes Lopes ${ }^{1}$, Tiago Barcelos Valiatti ${ }^{*}$, \\ Fabiana de Oliveira Solla Sobral1, Natália Faria Romãoํㅡ, \\ Graziella Pinetti Passoni ${ }^{2}$
}

\begin{abstract}
RESUMO
O objetivo deste trabalho foi verificar a eficiência do sistema de Análise de Perigos e Pontos Críticos de Controle (APPCC) implantado em indústria de laticínios através do monitoramento dos Pontos Críticos de Controle (PCC) identificados no plano APPCC para a produção do leite longa vida. Foram avaliados os processos produtivos de três caminhões. No PCC-1 realizaram-se testes de acidez titulável, alizarol, fosfatase alcalina, peroxidase e análise de resíduos de antibióticos. No PCC-2 foram analisadas a relação de tempo/temperatura da carta gráfica, bem como as análises de fosfatase alcalina e peroxidase com o intuito de avaliar a eficiência dos processos. No PCC-3 realizaram-se pesquisas de microorganismos aeróbios mesófilos em três caixas de leite longa vida após incubação de sete dias. Um total de 9 (100\%) das amostras indicaram acidez Dornic entre 0,14 e $0,16^{\circ} \mathrm{D} ; 9$ (100\%) apresentaram resultado negativo ao teste de alizarol; e 9 (100\%) mostraram resultado negativo para resíduos de antibióticos. As curvas gráficas mantiveram-se em seu tempo/temperatura correto, encontraram-se entre $74.1^{\circ} \mathrm{C}-74.2^{\circ} \mathrm{C} / 15-20 \mathrm{seg}$, o leite proveniente de cada caminhão apresentou resultado positivo para as enzimas fosfatase e peroxidase no leite cru, e após as pasteurizações o resultado foi negativo para enzima fosfatase e positivo para peroxidase. As amostras referentes a cada caminhão apresentaram resultado semelhante de $<1,0 \times 10^{\circ} \mathrm{UFC} / \mathrm{ml}$. Os resultados obtidos em cada PCC foram satisfatórios de acordo com a legislação pertinente. Os PCC estão dentro do estabelecido, demonstrando sua efetividade na aplicação do sistema APPCC na indústria de laticínios.
\end{abstract}

Palavras-chave: APPCC, Qualidade, Laticínios.

\footnotetext{
${ }^{1}$ Centro Universitário Luterano de Ji-Paraná, Ji-Paraná, Rondônia, Brasil.

2 Fundação Universidade Federal de Rondônia - Campus de Ji-Paraná - Secretaria do Campus, JiParaná, Rondônia, Brasil.

* Endereço para correspondência: Rua Roraima, 228 - Nova Ouro Preto - 76920-000, Ouro Preto do Oeste, Rondônia, Brasil. Telefone +55 (69) 99248-3512 / e-mail: gleh_gl@hotmail.com
} 


\section{Introdução}

Diante da importância do termo "qualidade", uma série de ferramentas foram criadas pela indústria alimentícia a fim de garantir que o produto final cumpra todos os requisitos exigidos e seja um alimento isento de riscos para o consumidor. Dentre as ferramentas, destaca-se a Análise de Perigos e Pontos Críticos de Controle (APPCC) (QUEIROZ \& ANDRADE, 2010).

Durante a cadeia produtiva em uma indústria alimentícia, o produto final pode ser comprometido devido a determinadas situações (perigos), sendo que é em razão dessas situações que entra o APPCC, que terá como função identificar os perigos bem como realizar a análise dos alimentos, buscando meios para corrigir os problemas a fim de garantir a qualidade de todo o processo (PAULA \& RAVAGNANI, 2009).

Diante de sua eficácia, é cada vez mais evidente que o APPCC é um diferencial na indústria de alimentos; prova disso é que os clientes mais exigentes, tanto os nacionais quanto os estrangeiros, consideram o APPCC como fator crucial no momento de fechar contratos (PAULA \& RAVAGNANI, 2011).

O modelo proposto pelo Codex Alimentarius para o sistema APPCC contém sete princípios básicos, em que primeiramente se analisam os perigos, sendo posteriormente determinados os Pontos Críticos de Controle (PCC), assim como o(s) limite(s) crítico(s), fazendo-se então necessárias as ações de monitoramento dos PCC, sendo então estabelecidas medidas corretivas para o seu controle. Por fim, são necessários procedimentos que verifiquem o funcionamento e o estabelecimento de um sistema com finalidade de registro e documentação.Destaca-se que os princípios descritos são aplicáveis durante todo o processo de produção (ALMEIDA, 1998).

Da sigla APPCC, os Pontos Críticos de Controle (PCC) consistem em etapas no qual um ou mais controles são aplicados, prevenindo ou 
eliminando os perigos (ALMEIDA, 1998). Salienta-se que, quando identificados, os PCC devem ser detalhadamente descritos e documentados, devendo ser evidenciados em um fluxograma contendo as letras que os representam $(\mathrm{Q}=$ químico; $\mathrm{B}=$ biológico; $\mathrm{F}=$ físico) (ALMEIDA, 1998; FIGUEIREDO \& COSTA, 2001).

Tendo em vista a importância do APPCC na cadeia de produção de alimentos e a ausência de estudos sobre o referido assunto na região central do estado de Rondônia, o presente estudo tem como objetivo verificar a eficiência do sistema APPCC implantado em indústria de laticínios do município de Ji-Paraná, RO, através do monitoramento dos Pontos Críticos de Controle (PCC).

\section{Material e métodos}

A indústria de laticínios avaliada funciona sob regime de Inspeção Federal (SIF), sendo responsável pelo recebimento de aproximadamente 450 mil litros de leite por dia, provenientes do próprio município e também de cidades vizinhas.

A visualização de uma árvore decisória contendo o fluxograma de produção fornecido pelo laticínio foi utilizada para destacar os PCC identificados no plano APPCC da indústria, sendo eles: recepção da matéria prima (PCC-1), pasteurização do leite (PCC-2) e esterilização do leite (PCC-3).

No decorrer do mês de setembro de 2014, foram avaliados os processo produtivos de três caminhões, sendo cada um dividido em três tanques com capacidade total de aproximadamente 30 mil litros de leite. Para cada caminhão, o leite foi processado e realizado o monitoramento dos PCC desde a recepção do leite na indústria até o produto final. 


\subsection{PCC-1: Recepção da matéria-prima}

A qualidade do leite cru refrigerado na propriedade rural e transportado a granel foi atribuída ao monitoramento do PCC-1 de recepção da matéria-prima. Para isso, as amostras foram coletadas em alíquotas de $500 \mathrm{~mL}$ de leite de cada tanque contido na plataforma de recepção da indústria e encaminhadas para realização das análises descritas abaixo, seguindo metodologia conforme recomendado por Brasil (2006).

A titulação por meio de graus Dornic e do teste em álcool a $76^{\circ} \mathrm{GL}$ (alizarol) foi empregada para avaliar os parâmetros de acidez. Na análise de acidez titulável, pipetou-se $11 \mathrm{~mL}$ da amostra em um béquer de $150 \mathrm{~mL}$ utilizando a pipeta graduada, adicionando 5 gotas de solução alcoólica fenolftaleína 1\% e titulado com a solução Dornic (Hidróxido de Sódio 0,1 N). Após o leite apresentar a coloração rosada, o resultado foi expresso em graus Dornic $\left({ }^{\circ} \mathrm{D}\right)$.Para o teste de alizarol utilizou-se um acídimitro de salut (pistola de alizarol), que dosou automaticamente $2 \mathrm{~mL}$ da solução alizarol a $76^{\circ} \mathrm{GL}$ e $2 \mathrm{~mL}$ de amostra de leite, tornando a prova mais rápida para a possível observação de cor. Após a viragem da cor o resultado foi expresso descrevendo a coloração do leite (INSTITUTO ADOLFO LUTZ, 2008).

A presença de fosfatase alcalina foi verificada utilizado um "kit" fosfatase alcalina da empresa DIASYS, seguindo seu procedimento com adição de $0,8 \mathrm{~mL}$ do reagente $1,0,2 \mathrm{~mL}$ do reagente 2 e $0,1 \mathrm{~mL}$ da amostra em tubo de ensaio, posterior inoculação de fita teste e observação da formação de cor na tira do kit fosfatase; a presença de coloração na tira indica positividade para fosfatase alcalina. Para a pesquisa de peroxidase, transferiu-se $10 \mathrm{ml}$ da amostra para um tubo de ensaio que foi aquecido em banho-maria a $45^{\circ} \mathrm{C}$ por cerca de 5 minutos, acrescentando-se $2 \mathrm{~mL}$ de solução hidroalcoólica de guaicol $11 \%$ ao tubo de ensaio, seguindo-se o acréscimo de 3 gotas da solução peróxido de hidrogênio a 3\%. Para o teste de resíduos de antibióticos foram utilizados testes rápidos (Kit Charm Quad1 BETA,TETRA, SULFA E QUINO), que permitem detecção de resíduos das drogas pertencentes às famílias: betalactâmicos, tetraciclinas, sulfonamidas e quinolonas no leite cru. Os testes foram colocados em uma placa aquecedora e realizada a leitura após 5 minutos através da interpretação do teste do Sistema EZ (HX0066-00205), que detecta positivo ou negativo para essas drogas no leite (INSTITUTO ADOLFO LUTZ, 2008). 


\subsection{PCC-2: Pasteurização do leite}

Para o controle das pasteurizações, foram realizados o acompanhamento visual por média de 60 minutos analisando a relação de tempo/temperatura da carta gráfica situada no pasteurizador, objetivando verificar se os limites críticos estavam entre $72-75^{\circ} \mathrm{C} / 15-20$ seg. Foram coletadas em frascos estéreis de $500 \mathrm{ml}$ amostras de leite submetido à pasteurização e encaminhadas para o laboratório de controle de qualidade, repetindo-se as análises das enzimas fosfatase alcalina e peroxidase para avaliar a eficiência dos processos, conforme estipulado por Brasil (BRASIL, 2011).

\subsection{PCC-3: Esterilização do leite}

Para o leite proveniente de cada caminhão, realizaram-se pesquisas de micro-organismos aeróbios mesófilos em três caixas de leite longa vida na embalagem fechada a $25-37^{\circ} \mathrm{C}$ durante sete dias. A contagem de aeróbios mesófilos foi realizada por semeadura de $1 \mathrm{~mL}$ da amostra em Plate Count Agar (PCA) para contagem, através de inoculação pour plate em diluições seriadas $10^{-1}$ a $10^{-5}$ seguida de incubação $36 \pm 1^{\circ} \mathrm{C}$ por 48 horas, conforme metodologia recomendada por Brasil (2003) e os resultados expressos em $\mathrm{UFC} / \mathrm{ml}$.

\section{Resultados e discussão}

$\mathrm{Na}$ Tabela 1 estão apresentados os resultados das análises físicoquímicas realizadas no leite cru recebido na plataforma industrial por cada caminhão e seus respectivos tanques, que são referentes ao monitoramento do PCC-1. 
Tabela 1. Resultado das análises físico-químicas do leite coletado dos caminhões-tanque na plataforma de recebimento da empresa.

\begin{tabular}{|c|c|c|c|c|}
\hline Rota & Tanque & Acidez ${ }^{\circ} \mathrm{D}$ & Alizarol & Antibiótico \\
\hline \multirow{3}{*}{ C. 1} & 1 & 0,14 & $76^{\circ} \mathrm{C}$ negativo & Negativo \\
\hline & 2 & 0,15 & $76^{\circ} \mathrm{C}$ negativo & Negativo \\
\hline & 3 & 0,15 & $76^{\circ} \mathrm{C}$ negativo & Negativo \\
\hline \multirow{3}{*}{ C. 2} & 1 & 0,16 & $76^{\circ} \mathrm{C}$ negativo & Negativo \\
\hline & 2 & 0,15 & $76^{\circ} \mathrm{C}$ negativo & Negativo \\
\hline & 3 & 0,15 & $76^{\circ} \mathrm{C}$ negativo & Negativo \\
\hline \multirow{3}{*}{ C. 3} & 1 & 0,15 & $76^{\circ} \mathrm{C}$ negativo & Negativo \\
\hline & 2 & 0,16 & $76^{\circ} \mathrm{C}$ negativo & Negativo \\
\hline & 3 & 0,15 & $76^{\circ} \mathrm{C}$ negativo & Negativo \\
\hline $\begin{array}{l}\text { Padrão IN } \\
62\end{array}$ & & 0,14 a $0,18^{\circ} \mathrm{D}$ & $76^{\circ} \mathrm{C}$ negativo & Negativo \\
\hline
\end{tabular}

C.1=Caminhão 1: C.2=Caminhão 2: C.3=Caminhão 3

Como observado na Tabela 1, constatou-se que 100\% das amostras estavam conforme a legislação vigente determina, podendo ser consideradas, assim, adequadas para a pasteurização do leite, o que permitiu dar prosseguimento às etapas de processamento (BRASIL, 2011).

Cullor (1993) destacou em seu trabalho que resíduos de antibiótico no leite deverão ser controlados no primeiro Ponto Crítico de Controle (PCC), que é a recepção do leite cru no laticínio.

No presente estudo, o resultado (100\%) negativo para resíduos de antibiótico em todos os tanques de cada caminhão que foi monitorado no PCC-1 da indústria foi fundamental para a qualidade do processamento. $\mathrm{O}$ leite recebido pela empresa poderia representar perigo químico decorrente do envio do leite de animais em tratamento com resíduo de antibiótico (GUIMARÃES, 2012).

No entanto, resultados positivos foram encontrados por Barros, Jesus e Silva (2001), que estudando resíduos de antibióticos em leite de vaca obtiveram resultados superiores aos encontrados neste estudo. O mesmo foi 
encontrado por Ferreira et al. (2014), porém em ambos os estudos não havia relatos da aplicação do plano APPCC.

A análise da carta gráfica acompanhada de forma visual referente ao monitoramento do PCC-2 durante a etapa de pasteurização do leite revelou que as curvas gráficas mantiveram-se em seu tempo/temperatura corretos, não ultrapassando os limites críticos. Encontraram-se entre $74.1^{\circ} \mathrm{C}$ $74.2^{\circ} \mathrm{C} / 15$-20 seg., conforme estabelece a legislação (BRASIL, 2011).

Os limites da temperatura e tempo de aquecimento $\left(72^{\circ}\right.$ a $75^{\circ} \mathrm{C}$ por 15 a 20 segundos) na pasteurização devem ser constantemente analisados, visto que essa etapa é um PCC (BRASIL, 2011). Na pasteurização os equipamentos devem ser projetados de modo a permitir o monitoramento e o controle de temperatura (GUIMARÃES, 2012).

Ainda no que diz respeito ao PCC-2, na Tabela 2 estão descritos os resultados obtidos nas análises físico-químicas de atividade enzimática de fosfatase alcalina e peroxidase antes e após a pasteurização do leite

Tabela 2. Resultado das atividades enzimáticas do leite cru e do leite pasteurizado.

\begin{tabular}{|c|c|c|c|c|c|}
\hline \multirow[b]{2}{*}{ Rota } & \multicolumn{2}{|c|}{ Leite cru } & \multicolumn{3}{|c|}{ Leite pasteurizado } \\
\hline & Tanque & Fosfatase & Peroxidase & Fosfatase & Peroxidase \\
\hline \multirow{3}{*}{ C. 1} & 1 & Positivo & Positivo & Negativo & Positivo \\
\hline & 2 & Positivo & Positivo & Negativo & Positivo \\
\hline & 3 & Positivo & Positivo & Negativo & Positivo \\
\hline \multirow{3}{*}{ C. 2} & 1 & Positivo & Positivo & Negativo & Positivo \\
\hline & 2 & Positivo & Positivo & Negativo & Positivo \\
\hline & 3 & Positivo & Positivo & Negativo & Positivo \\
\hline \multirow{3}{*}{ C. 3} & 1 & Positivo & Positivo & Negativo & Positivo \\
\hline & 2 & Positivo & Positivo & Negativo & Positivo \\
\hline & 3 & Positivo & Positivo & Negativo & Positivo \\
\hline
\end{tabular}

Não foram observadas falhas nas pasteurizações do leite, pois a Tabela 3 evidencia a eficiência no processo de pasteurização. 
Vale destacar que imediatamente após a pasteurização devem ser realizadas as pesquisas das enzimas peroxidase e fosfatase para verificar a eficiência do processo, sendo que o leite pasteurizado deve apresentar-se negativo para fosfatase alcalina e positivo para peroxidase (BRASIL, 2011).

Como constatado o resultado positivo para a enzima peroxidase após todas as etapas de pasteurização que foram monitoradas, evidencia-se que os leites não foram superaquecidos, portanto não tiveram sua composição alterada. O superaquecimento pode ocorrer em duas situações distintas; na primeira, em decorrência da falta de qualidade da matéria prima, em que o superaquecimento vai garantir durabilidade e os parâmetros microbiológicos; já na segunda, ocorre devido a alguma falha no processo de controle da temperatura do pasteurizador (TAMANINI et al., 2007).

Arquelau (2013), ao analisar por meio do monitoramento dos Pontos Críticos de Controle uma usina piloto que possui o sistema APPCC implantado para controle de qualidade dos seus produtos, relata resultados semelhantes aos encontrados no PCC-2 desta pesquisa, pois o autor mostra em seu trabalho que a eficiência da pasteurização foi observada pela curva gráfica do pasteurizador, com o tempo/temperatura entre $72-75^{\circ} \mathrm{C} / 15$-20seg, respeitado rigorosamente, e o teste de enzimas no leite pasteurizado, que obteve resultado positivo para peroxidase e negativo para fosfatase alcalina, como preconizado pela legislação (BRASIL,2011).

Carvalho (1998), ao controlar a eficácia da pasteurização, constatou que todas as amostras estavam dentro dos padrões para fosfatase alcalina. No caso da peroxidase, $50 \%$ das amostras apresentam resultados negativos, demonstrando falha no processamento, uma vez que não foi obedecido o binômio tempo/temperatura estipulado pela legislação. Já Rosa-Campos et al. (2011), ao desenvolver um estudo em Brasília, verificou que todas as marcas de leite analisadas continham amostras fora do padrão permitido, demonstrando assim a necessidade de programas que garantam a qualidade do produto ao consumidor, como o plano APPCC. 
Os resultados da contagem padrão em placas de aeróbios mesófilos em unidades formadoras de colônias (UFC/mL), realizado de forma aleatória nas caixas de leite longa vida, revelaram que todas as amostras não apresentaram contagem de microrganismos acima do limite de deteç̧ão do teste (<1,0 x 100), conforme o estabelecido (BRASIL, 1996).

Nesta pesquisa, resultados satisfatórios foram encontrados no monitoramento do PCC-3, podendo-se afirmar que as características mínimas foram atendidas em todas as amostras analisadas, tendo-se a certeza de eliminação de todos os microrganismos e da qualidade do produto final. Yoshisuki \& Cogo (2014), ao analisarem bactérias aeróbias mesófilas em leite longa vida, observaram resultados semelhantes aos aqui encontrados.

Domareski et al. (2010), ao avaliarem leite longa vida, verificaram que $37,5 \%$ das amostras brasileiras, $62,5 \%$ das amostras paraguaias e $12,5 \%$ das argentinas estavam contaminadas por microrganismos aeróbios mesófilos acima do recomendado (100 UFC/ml) para esse tipo de leite.Já Bersot et al. (2010) observaram resultados opostos ao do presente estudo, pois $24 \%$ das amostras analisadas de leite submetido à ultra-alta temperatura estavam contaminadas por aeróbios mesófilos.

Os resultados obtidos em todos os Pontos Críticos de Controle (PCC) se fizeram satisfatórios de acordo com a legislação vigente (BRASIL1996; BRASIL, 2011), apontando 100\% de eficiência do plano de Análise de Perigos e Pontos Críticos de Controle (APPCC) na indústria de laticínios avaliada.

Estudos como o de Al-Saed et al. (2012) constataram uma significativa melhoria na qualidade microbiológica de Jameed (produto lácteo fermentado) após a aplicação do sistema do PCC. Já Tomasevic et al. (2016), ao compararem41 meses antes e 43 meses depois estabelecimentos de varejo e de processamento de carne após a implantação do APPCC, 
observaram considerável diminuição de contaminação microbiológica, demonstrando o quanto essa ferramenta é eficaz.

\title{
4 Conclusão
}

Por meio deste estudo foi possível monitorar os PPCs durante a cadeia produtiva do leite, desde a recepção da matéria-prima até a obtenção do produto final, mostrando que os Pontos Críticos de Controle estão sendo controlados sistematicamente em todas as etapas da linha de produção, e diariamente são exercidos procedimentos no sentido de evitar, reduzir ou eliminar a presença dos possíveis perigos, evidenciando a eficácia na aplicação do sistema de APPCC na indústria de laticínios do município estudado.

\section{Monitoring of critical control points applied in the processing of long life milk in the dairy industry of ji-paraná}

\begin{abstract}
To verify the efficiency of the Hazard Analysis and Critical Control Points (HACCP) system implemented in the dairy industry by monitoring the Critical Control Points (CCPs) identified in the HACCP plan for the production of long-life milk. The production process of three trucks was evaluated in the PCC-1 titratable acidity tests, alizarol, alkaline phosphatase, peroxidase and antibiotic residue analysis. In PCC-2, the time / temperature relationship of the chart was analyzed, as well as the alkaline phosphatase and peroxidase analyzes in order to evaluate the efficiency of the processes. In PCC-3, we performed surveys of aerobic mesophilic microorganisms in three boxes of long-life milk after incubation for seven days. A total of $9(100 \%)$ of the samples indicated Dornic acidity between 0.14 and $0.16^{\circ} \mathrm{D}, 9(100 \%)$ presented negative results to the alizarol test and $9(100 \%)$ showed negative results for antibiotic residues. The graphical curves were maintained at their correct temperature / time, between $74.1^{\circ} \mathrm{C}-74.2^{\circ} \mathrm{C} / 15-20 \mathrm{sec}$, the milk from each truck presented a positive result for the enzymes phosphatase and peroxidase in the raw milk, after the pasteurisations. Result was negative for enzyme phosphatase and positive for peroxidase. Samples from each truck had a similar result of $<1.0 \times 10^{\circ} \mathrm{CFU} / \mathrm{ml}$. The results obtained in each $\mathrm{CCP}$ were satisfactory according to the relevant legislation. The CCPs are within the established, demonstrating their effectiveness in the application of the HACCP system in the dairy industry.

Keywords: HACCP, Quality, Dairy.
\end{abstract}




\section{Referências Bibliográficas}

ALMEIDA, C. R. O sistema HACCP como instrumento para garantir a inocuidade dos alimentos. Higiene Alimentar. v. 12, n. 53, p. 12-2, 1998.

AL-SAED, A.K.; AL-GROUM, R.M.; AL-DABBAS, M.M. Implementation of hazard analysis critical control point in jameed production. Food Science and Technology International. v. 18, n. 3, p. 229-239, 2012.

ARQUELAU, P. B. F. Avaliação da qualidade físico-química de leites pasteurizados comercializados no Distrito Federal e elaboração de um derivado lácteo. 2013. 49f. Trabalho de conclusão de curso (Graduação em Farmácia). Ceilândia: Universidade de Brasília, 2013.

BARROS, G. M. S.; JESUS, N. M.; SILVA, M. H. Pesquisa de resíduos de antibióticos em leite pasteurizado tipo C, comercializado na cidade de Salvador. Revista Brasileira de Saúde e Produção Animal. v. 3, n. 2, p. 69-73, 2001.

BERSOT, L. S.; GALVÃO, J. A.; RAYMUNDO, N. K. L.; BARCELLOS, V. C.; PINTO, J. P. A. N.; MAZIERO, M. T. Avaliação microbiológica e físico-química de leites UHT produzidos no Estado do Paraná - Brasil. Semina: Ciências Agrárias. v. 31, n. 3 , p. $645-652,2010$.

BRASIL. Agência Nacional de Vigilância Sanitária. Regulamento técnico sobre padrões microbiológicos para alimentos. Resolução RDC n ${ }^{\circ} 12$ de 02 de janeiro de 2001. Diário Oficial [da] República Federativa do Brasil, Brasília, 10 jan. 2001. Seção 1, p. 45-53.

.Manual Genérico de Procedimento para APPCC em Indústrias de Produtos de Origem Animal. Diário Oficial [da] República Federativa do Brasil, Brasília, 16 mar. 1998. Seção 1, p.24. 
Ministério da Agricultura e do Abastecimento. Portaria nº 146, de 07 de março de 1996. Aprova os Regulamentos Técnicos de Identidade e Qualidade dos Produtos Lácteos. Diário Oficial [da] República Federativa do Brasil, Brasília, 11 mar. 1996. Seção 1, p. 3977.

Ministério da Agricultura Pecuária e Abastecimento. Instrução Normativa $n^{\circ} 62$ de 26 de agosto de 2003. Métodos Analíticos Oficiais para Análises Microbiológicas para Controle de Produtos de Origem Animal e Água. Diário Oficial [da] República Federativa do Brasil, Brasília, 18 set. 2003. Seção 1, p.14.

Ministério da Agricultura, Pecuária e Abastecimento. Instrução Normativa n.62, de 29 de dezembro de 2011. Aprova o Regulamento Técnico de Produção, Identidade e Qualidade do Leite tipo A, o Regulamento Técnico de Identidade e Qualidade de Leite Cru Refrigerado, o Regulamento Técnico de Identidade e Qualidade de Leite Pasteurizado e o Regulamento Técnico da Coleta de Leite Cru Refrigerado e seu Transporte a Granel, em conformidade com os anexos desta Instrução Normativa. Diário Oficial [da] República Federativa do Brasil, Brasília, 30 dez. 2011. Seção 1, p. 6-11.

Ministério da Agricultura Pecuária e Abastecimento. Portaria n ${ }^{\circ} 46$, de 10 de fevereiro de 1998.

- Ministério da Agricultura, Pecuária e Abastecimento. Instrução Normativa $\mathrm{n}^{\circ} 68$ de 12 de Dezembro de 2006. Normas para Métodos Analíticos Oficiais Físico-Químicos, para Controle de Leite e Produtos Lácteos. Diário Oficial [da] República Federativa do Brasil, Brasília, 14 dez. 2006. Seção 1, p.8.

CARVALHO, M. G. X. Características físico-químicas, biológicas do leite de cabra processado em micro usinas da Região da Grande São Paulo - SP. 1998. [tese]. São Paulo: Faculdade de Medicina Veterinária e Zootecnia, Universidade de São Paulo. 
CODEX ALIMENTARIUS. Food hygiene basic texts. Ed. 2.77 p. 2002. Disponível em: ftp://ftp.fao.org/docrep/fao/005/Y1579e/Y1579e.pdf>. Acesso em: 14 mai. 2017.

CULLOR, J. S. Antibiotic residue test for mammary gland secretion. The Veterinary Clinics of North America: Food Animal Practice. v. 9, n. 3, p. 609620, 1993.

DOMARESKI, J. L.; BANDIERA, N. S.; SATO, R. T.; ARAGON, L. C.; SANTANA, E. H. W. Avaliação físico-química e microbiológica do leite UHT comercializado em três países do Mercosul (Brasil, Argentina e Paraguai). Archivos Latinoamericanos de Nutrición. v. 60, n. 3, p. 261-269, 2010.

FERREIRA, A.P.; MACEDO, L. S. O; ROCHA, T. S.; RAMOS, L.S, N. Resíduos de antibióticos em leite in natura utilizado para processamento em laticínio localizado no município de Teresina-Piauí. Acta Tecnológica. v. 9, n. 1, p. 9-12, 2014.

FIGUEIREDO, V. F.; COSTA, N. P. L. O. Implantação do HACCP na indústria de alimentos. Gestão \& Produção. v. 8, n. 1, p.100-111, 2001.

GUimarães, T. G. Determinação dos Pontos Críticos de controle em Indústria de Laticínios. 2012. 67p. Trabalho de conclusão de curso (graduação em Medicina Veterinária) - Universidade Federal do Rio Grande do Sul, Porto Alegre, 2012.

Instituto Adolfo Lutz (São Paulo). Métodos físico-químicos para análise de alimentos. Coordenadores Odair Zenebon, Neus Sadocco Pascuet e Paulo Tiglea São Paulo: Instituto Adolfo Lutz, p. 1020, 2008.

PAULA, S. L.; RAVAGNANI, M. A. S. S. Lógica Fuzzy como Ferramenta de Decisão na Identificação dos Perigos Significativos e Medidas Preventivas de Controle do Sistema APPCC (Análise de Perigos e Pontos Críticos de Controle). In: CIBIA VII, Colômbia, 2009. CD - RQM. 
PAULA, S. L.; RAVAGNANI, M. A. S.S. Sistema APPCC (Análise de Perigos e Pontos Críticos de Controle) de acordo com a NBR ISO 22000. Revista Tecnológica Maringá, v. 20, 2011, p. 97-104, 2011.

QUEIROZ, V. M.; ANDRADE, H. V. Importância das ferramentas da qualidade BPF/APPCC no controle dos perigos nos alimentos em um laticínio. Cadernos de Pós-graduação da fazu. v. 1, 2010.

ROSA-CAMPOS, A. A.; ROCHA, J. E. S.; BORGO, L. A.; MENDONÇA, M. A. "Avaliação físico-química e pesquisa de fraudes em leite pasteurizado integral tipo C" produzido na região de Brasília, Distrito Federal. Revista Instituto Laticínios Cândido Tostes. v. 66, n. 379, p. 30-34, 2011.

TAMANINI R, SILVA. L.C, MONTEIRO, A. A.; MAGNANI, D. F.; BARROS, M. A. F.; BELOTI, V. Avaliação da Qualidade Microbiológica e dos Parâmetros Enzimáticos da Pasteurização de Leite Tipo C Produzido na Região do Norte do Paraná. Revista de Ciências Agrárias. v. 28, n. 3, p. 449-454, 2007.

TOMASEVIC, I; KUZMANOVIĆ, J; ANĐELKOVIĆ, A.; SARAČEVIĆ, M;/ STOJANOVIĆ. M. M.; DJEKIC, I. The effects of mandatory HACCP implementation on microbiological indicators of process hygiene in meat processing and retail establishments in Serbia. Meat Science. v. 114, p. 54-57, 2016.

YOSHISUKI, P. Y; COGO, L. L. Qualidade microbiológica do leite comercializado no município de Curitiba, Paraná. Visão Acadêmica. v. 15, n. 1, p. 98-106, 2014. 